\title{
DROIT ET SEMIOTIQUE: LA COHERENCE NARRATIVE
}

\author{
Sylvie André
}

In this article, Professor André considers the nature of legal rules, their methods of creation and their interpretation and application. The role of modern narrative theory in answering these perennial questions is explored and two conclusions are reached: first, the classic explanations of legitimacy that underpin reasoning in the social sciences are increasingly losing ground; and, secondly, contemporary literary accounts based on the reasons for this loss of ground provide a strong challenge to narratives of coherence that are closely linked to Western culture. The existing model of knowledge does not correspond to the reality of contemporary society; the rules and principles that even today are still regarded as universal are seen by a large fraction of the human race as relative and cultural. Insights from narrative theory show that the perennial law questions must now be revisited with a new perspective.

\section{INTRODUCTION}

Les philosophes du droit s'interrogent sur la nature de la norme juridique, sur ses modalités de création ainsi que sur son interprétation et son application. Un article de Patrick Nérhot, notamment, ${ }^{1}$ propose d'utiliser le concept sémiotique de narrativité pour répondre à ces interrogations.

Il s'attache tout d'abord à faire la démonstration que rien ne permet d'avancer à coup sûr que des principes "naturels" préexistent à la formulation de la loi qui n'en serait que la transcription. Nérhot met au contraire en évidence l'historicité de la règle de droit, qui n'a rien d'intemporel. Science sociale, il est évident que le droit évolue par la jurisprudence ou par l'interprétation en ce qui concerne la Common Law.

* Ancienne élève de l'ENA, Université Paris III.

1 Patrick Nérhot "L'Interprétation en sciences juridiques. La notion de cohérence narrative" (1990) 111 Revue de synthèse 299. 
En outre, la création même de la règle juridique repose selon lui sur des opérations logiques qui ne sont pas réductibles à une logique mathématique. En effet, la science juridique ne saurait faire siens les principes philosophiques qui sous-tendent les modèles des sciences exactes. Ceux-ci utilisent des présupposés qui peuvent se résumer à quatre termes: la réalité est objective, déterminée, globale et une. ${ }^{2}$ Les sciences exactes recherchent une vérité atemporelle, intangible et universelle, "a-humaine". Pour la science juridique, une telle vérité est hors d'atteinte et sans doute dépourvue d'intérêt, puisque c'est de l'homme et de la société qu'il s'agit.

Nérhot propose alors l'hypothèse "de la cohérence narrative comme fondement du raisonnement juridique". ${ }^{3}$ La cohérence narrative est ainsi posée comme une modélisation de l'expérience humaine, irréductible à la modélisation mathématique. Cette modélisation viserait à donner sens à une réalité plurielle, mouvante, diverse mais humaine. Cette conception du récit en tant que modèle original du savoir alimente le phénomène du tournant narratif ou "narrative turn" qui imprègne les sciences humaines et sociales anglo-saxonnes depuis vingt ans: ${ }^{4}$

In the last twenty years this transformation has put the problematics of narrative at the center of not only historical, ethnographical, and psychoanalytical thought, but also juridical, political, and even medical, thus endowing narrative theory with the status of a new paradigm for knowledge theory.

Curieusement, dans l'article de Nérhot, l'expression 'cohérence narrative' n'est jamais définie pour elle-même mais toujours par rapport à son utilisation par l'épistémologie d'une autre science humaine: l'Histoire. Nérhot construit son argumentation sur un parallélisme étroit entre les théories de Paul Ricœur, Paul Veyne et la recherche de normes en sciences juridiques. En fait, tout se passe comme si réfutant la logique purement mathématique en tant que source de la loi, de même qu'il réfute, comme nous l'avons dit, les théories d'une "découverte" de principes transcendants, il ne lui restait que l'hypothèse de la cohérence narrative empruntée à la science historique pour rendre compte du raisonnement juridique: "[1]e juriste en d'autres termes, se trouve bien dans la situation d'un historien ou réciproquement, si l'on veut. La méthode historique est donc centrale". $5 \mathrm{Ce}$ parallélisme ne fait aucune difficulté sans doute pour tout ce qui concerne l'interprétation des textes au sens de la jurisprudence. De même qu'un cas nouveau peut entraîner un revirement de jurisprudence, de même un événement, de nouveaux documents découverts peuvent donner lieu à une relecture de l'Histoire. Sinon, c'est bien l'histoire des décisions juridiques passées qui sert à éclairer les décisions présentes. Mais de telles constatations ne justifient pas totalement l'utilisation

2 Giorgio Israël "Modèle récit ou récit modèle" dans Jean-Yves Grenier, Claude Grignon et Pierre-Michel Menger (dir) Le Modèle et le Récit (Editions de la Maison des sciences de l'homme, Paris, 2001) 365, 378379.

3 Nérhot, précité n 1, 306.

4 Sylvie Patron "Describing the Circle of Narrative Theory: A Review Essay" (2005) 39 Style 479, 479.

5 Nérhot, précité n 1, 320. 
d'un terme qui relève du champ de la sémiotique - le récit ou narrative - et pas seulement de l'analyse historique. C'est à justifier ce recours, tout à fait pertinent sinon clairement défini, à la définition sémiotique du récit que nous voudrions nous employer ici.

\section{IDEOLOGIES VERSUS METARECITS}

Une dimension de la démonstration de Nérhot évoquée ci-dessus ne fait pas réellement difficulté, comme nous l'avons dit. C'est pourquoi nous l'envisageons en premier lieu et nous allons nous efforcer de la relier à certaines théories du récit, en dépassant la simple question de l'historicité: 6

Les facteurs extra-législatifs de production du droit, sont des facteurs historiques qui vont déterminer le sens même des énoncés, présentés comme techniques. ... Un énoncé, aussi technique soit-il, et selon les périodes historiques considérées, pourra recouvrir des sens bien différents.

En effet, il est facile de comprendre l'importance de facteurs sociologiques et idéologiques pour l'interprétation de la loi et même pour sa production: faut-il rappeler l'exemple de Jean Valjean, héros des Misérables de Victor Hugo, condamné au bagne pour avoir volé du pain? Une telle protection du principe de propriété nous apparaît aujourd'hui dans toute son outrance inacceptable. Mais si le lien du raisonnement juridique à l'histoire des idées paraît incontestable, comment introduire ici la cohérence narrative, autrement dit l'importance du récit en tant que modèle du savoir?

En fait les philosophes contemporains ont fourni aux sciences humaines un cadre épistémologique extrêmement productif, celui des "grands récits" ou métarécits, qui pourrait être fort utile ici. Cette notion est développée par Jean-François Lyotard dès 1979 dans La Condition postmoderne, ${ }^{7}$ qui était à l'origine un rapport commandé par le gouvernement canadien sur les implications de la nouvelle société de l'information. S'interrogeant sur les conditions du savoir Lyotard postule l'existence à l'époque moderne de deux visions philosophiques implicites, que l'on ne peut définir dans leur totalité mais qui se réalisent partiellement dans tous les récits produits par le savoir. L'une est la vision positiviste qui recherche le fonctionnement optimum du système social, l'autre, critique, qui s'interroge sur les buts légitimes d'une société. Cette légitimation n'est affaire que de discours, de pratique langagière et d'interaction communicationnelle: elle ne peut donc être fournie par les sciences "exactes". Lyotard va plus loin et postule que la science ne peut se légitimer elle-même sinon par une logique de puissance et de performativité. Or, jusqu'ici cette logique n'a jamais été pleinement acceptée par les sociétés humaines qui légitiment le pouvoir par des règles et des valeurs morales, par une conception de l'être humain et de ses finalités. Ainsi donc, à l'époque

6 Ibid, 303.

7 Voir Jean-François Lyotard La condition postmoderne. Rapport sur le savoir (Les Editions de Minuit, Paris, 1979). 
moderne, de grands récits de légitimation tels la philosophie allemande du 19ième siècle ou encore le marxisme ont donné aux êtres humains des raisons de vivre et de développer la connaissance. Le savoir humain inclut sciences, connaissances, règles: "le savoir est ce qui rend quelqu'un capable de proférer de 'bons' énoncés dénotatifs, mais aussi de 'bons' énoncés prescriptifs, de 'bons' énoncés évaluatifs...". 8 Seul le récit peut lier toutes les dimensions du savoir et rendre compte de leur cohérence.

Cette notion de "métarécits" a été développée par la suite, par Marc Augé, ethnologue, par exemple, ou encore Alain Badiou, philosophe engagé. Marc Angenot, historien et professeur de littérature, dans son ouvrage sur Les grands récits militants $d u X I X^{\circ}$ et $X X^{\circ}$ siècles, donne une définition simple des "grands récits": 9

Convenons d'appeler "grands récits", les complexes idéologiques qui se sont chargés de procurer aux modernes une herméneutique historique totale, balayant les horizons du passé, du présent et de l'avenir-

le programme "utopique" qu'ils comportent y formant la "pars construens" d'une édification cognitive.

Comme nous le disions, ce discours "total" peut difficilement être connu dans sa globalité. On peut l'inférer des petits récits qui participent de ce discours. Ainsi, selon Angenot, le métarécit de la modernité est-il celui d'une histoire des hommes à la fois intelligible et maitrisable qui aboutira à révéler l'essence de l'humain. ${ }^{10}$ Nous comprenons dès lors que la structure de sens ne vient pas de la chronologie elle-même mais bien de la structure du récit avec un début, une fin, un héros qui est l'humanité, posant des valeurs par ses actes. Augé, dans son ouvrage Fictions fin de siècle, ${ }^{11}$ reprend cette conception de l'élaboration constante de récits reposant sur une idée implicite qui est de donner sens au lien social par le discours. Et nous voyons l'apport du modèle du récit par rapport au concept plus ancien d'idéologie: il met l'accent sur une cohérence, un sens qui se construit sur la ligne du temps humain mais qui n'est pas réductible au déroulement chronologique. Le juriste est donc pris dans ces métarécits, non seulement au niveau de l'interprétation de la loi mais aussi au niveau de sa création, car ces métarécits donnent forme à notre réflexion à une profondeur qui n'est pas celle de la conscience claire.

$8 \quad$ Ibid, 37

9 Marc Angenot Les grands récits militants des XIX ${ }^{\circ}$ et $X X^{\circ}$ siècles (L'Harmattan, Paris, 2000) 7.

10 La relation de ce métarécit de la modernité avec le Livre des livres de la civilisation occidentale, la Bible, est soulignée par Northrop Frye dans l'Ecriture profane. Essai sur la structure du romanesque (Circé, Paris, 1999) 190. Il y explique comment le mythe de la création du monde par un Dieu-Père a influencé la structure de la totalité des récits profanes. Ce mythe fait de la réalité "quelque chose d'humainement intelligible, en nous fournissant un monde qui commence et qui se termine dans le temps". Rappelons que la Bible va de la Genèse à l'Apocalypse.

11 Voir généralement Marc Augé Fictions fin de siècle (Fayard, Paris, 2000). 


\section{MISE EN EVIDENCE DE LOIS HISTORIQUES ET CREATION DE LA NORME JURIDIQUE}

Reste la question centrale. Comment parler de cohérence narrative au niveau proprement dit de la création de la norme? Comme nous le disions, c'est le parallèle avec la science historique qui fournit des arguments à Nérhot. Il s'appuie sur elle pour démontrer l'association inévitable de la chaîne causale et du temps en sciences humaines de même que de la nécessaire présence de l"'imagination" dans les récits du savoir. Il se réfère plus particulièrement aux analyses épistémologiques des historiens contemporains, comme nous l'avons dit en introduction. Guidés au départ par une recherche de scientificité supérieure, les historiens en ont précisé les limites en sciences humaines. L'école historique du positivisme logique par exemple a publié en anglais une réflexion abondante sur ses principes épistémologiques. Raymond Aron en résume ainsi l'idée force: "[i]l n'y a pas une réalité historique, toute faite ... qu'il conviendrait simplement de reproduire avec fidélité". ${ }^{12}$ Face à ce constat, comment éviter l'universel bavardage et comment définir des objets et des méthodes permettant d'atteindre à une forme de vérité? Cette école a donc tenté de faire de l'Histoire une science à part entière en mettant en évidence des lois, raison pour laquelle Ricœur la dénomme école du modèle "nomologique". En fait, partant du principe que "les lois générales ont des fonctions tout à fait analogues en histoire et dans les sciences naturelles", 13 quelqu'un comme Carl G Hempel pose que rien ne distingue un événement humain d'un phénomène physique. Tous deux obéissent à des lois conçues comme des "régularités vérifiées" ${ }^{14}$ et il faut se garder d'accorder une valeur proprement épistémologique à des procédés qui s'autoriseraient du titre d'empathie, de compréhension ou d'interprétation. ${ }^{15}$ Autrement dit, il importe que la personnalité de l'historien n'intervienne en rien dans l'explication. Toutefois, ces thèses extrêmes ont dû être sérieusement nuancées, tout en reconnaissant l'apport épistémologique du modèle nomologique. Il a fallu admettre par exemple que les lois mises en évidence étaient davantage des corrélations à haute fréquence que des relations invariables.

En effet, contrairement au physicien qui, par la recherche de lois vise à réduire la contingence, l'historien pour sa part veut d'abord expliquer les événements contingents, humains. ${ }^{16}$ On a aussi reproché aux lois qu'il utilise de n'être que la mise en évidence d'une intelligibilité préétablie par des modèles culturels tels que le récit par exemple. L'historien ne découvrirait pas des lois mais les

12 Raymond Aron cité dans l'ouvrage de Paul Ricœur Temps et récit, Vol I. L'intrigue et le récit historique (Seuil, Paris, 1991) 175

13 Carl G Hempel "The Function of General laws in History" (1942) 39 The Journal of Philosophy 35, 35 (traduction de l'auteur).

14 Ricœur, précité n 12, 202-203; Hempel, précité n 13, 35-38.

15 Hempel, précité n 13, 44-45.

16 Ricœur, précité n 12, 274. 
emploierait. On a par ailleurs opposé à cette école la sélectivité des explications retenues: en effet, dans un ensemble difficilement dénombrable d'événements antécédents on va désigner l'un d'entre eux comme cause principale d'un événement postérieur. De plus, l'historien délimite son champ d'étude, préjugeant en ceci d'une intelligibilité à découvrir, mais ce trait est aussi vrai pour les sciences "dures". Enfin, la loi en Histoire ne permet pas réellement de rendre compte des événements qui vont se produire, l'explication est en effet rarement reproductible en l'état contrairement à ce qu'exige la science en temps normal. On voit dès lors la proximité épistémologique des lois mises à jour en Histoire avec la création des normes juridiques. Mais en quoi une logique causale, certes non strictement scientifique, relève-t-elle du récit? Il faut poursuivre l'analyse des historiens pour le comprendre.

Les tenants du modèle nomologique ont donc dû modifier leurs positions de telle sorte que plutôt que de mettre en évidence la ressemblance de l'explication historique avec l'explication objective, ils en ont au contraire montré la spécificité. Ainsi, par exemple, dire que la cause principale est déterminée implicitement par ce que l'on veut démontrer, ou encore qu'en fonction de la finalité poursuivie, un choix de causalité va être fait, explique qu'il puisse exister plusieurs interprétations d'un même événement, l'enchaînement causal étant chaque fois impeccable. Comment ne pas voir que l'on rejoint ici la pluralité des récits dans une société donnée, dont le critère majeur d'acceptance est qu'ils ne soient pas en contradiction flagrante avec l'horizon d'attente du récepteur, pour utiliser les termes de Jauss, repris par Ricœur? ${ }^{17}$

A travers la lecture critique d'auteurs tels que Hayden White ${ }^{18}$ ou Paul Veyne ${ }^{19}$ pour la France, Ricœur précise ce que l'Histoire doit au récit et il s'appuie pour cela sur les dernières études connues relatives à la nature du récit. S'il devient désormais acceptable de réhabiliter le récit historique, c'est que le récit en tant que tel a été réévalué en fonction de la reconnaissance de ses ressources en intelligibilité. ${ }^{20}$ Ainsi, White s'appuie-t-il sur les travaux de Northrop Frye relatifs à la mise en intrigue. Selon lui, histoire et philosophie de l'histoire sont toujours étroitement unies, et sont en parie le reflet de la personnalité de l'historien. L'Histoire est un genre mêlé où l'explication peut se faire par des lois, des causes, des intentions ou encore des interventions humaines. De même dans la

17 Voir sur ce sujet F Jameson Archéologies du futur (Max Milo Editions, Paris, 2007) 163, qui fait une analyse très proche de celle-ci:

Il me paraît plus plausible ... de considérer la causalité comme une catégorie essentiellement narrative. ... [A]vec l'augmentation de la masse des choses que la recherche historique incline à considérer comme déterminantes ... chacune de ces choses ... dicte à son tour un nouveau récit historique, une manière neuve de raconter l'histoire... .

18 Voir Hayden White Metahistory: The Historical Imagination in Nineteenth-Century Europe (The Johns Hopkins University Press, Baltimore, 1973).

19 Voir Paul Veyne Comment on écrit l'histoire (Seuil, Paris, 1971).

20 Ricœur, précité n 12, 217. 
confection de la décision juridique, "[1]a société est comprise à partir de principes explicatifs de nature rationnelle, intentionnelle et psychologique et de type également causal ou probabiliste", ${ }^{21}$ puisque cette décision ne peut se fonder ni sur l'observation, ni sur une logique purement mathématique. Il en va de même dans le récit. Comprendre l'Histoire et comprendre une histoire obéissent aux mêmes exigences d'un lecteur qui doit trouver les récits acceptables et de manière corollaire cohérents, ce que certains auteurs ont désigné sous le vocable de followability: "l'historien en tant qu'écrivain, s'adresse à un public susceptible de reconnaître les formes traditionnelles de l'art de raconter". ${ }^{22}$ Mais il faut pousser notre analyse plus avant si nous voulons bien comprendre la place de la cohérence narrative dans l'élaboration de la règle juridique. Cela revient à poser que le récit n'est pas pure succession chronologique, il n'est jamais reproduction d'un ordre qui lui serait extérieur et qui serait l'ordre de succession des événements. Cette intelligibilité du récit rapproche Histoire et Droit.

\section{COHERENCE NARRATIVE, INTRIGUE ET MODELE NARRATIF}

En fait, s'appuyant sur les analyses de White, Ricœur insiste sur l'importance, corroborée par Veyne, de la mise en intrigue dans l'écriture de l'Histoire: " [1]'histoire n'est que construction et compréhension d'intrigues". ${ }^{23}$ Rappelons ici que l'Ecole des Annales a fait un sort à l'Histoire événementielle, détachant ainsi la structure narrative de la biographie individuelle de personnages remarquables et le sens, de volontés individuelles d'action sur le monde. Selon Ricoeur, en privilégiant l'étude des phénomènes de longue durée, l'Ecole des Annales et Fernand Braudel, son représentant le plus connu, ont effectué une médiation entre l'événement et la structure. La quête d'intelligibilité qui est au cœur de tout récit permet d'unir faits contingents et exigence d'ordre, épisode isolé et configuration signifiante, discordance de l'événement et concordance structurelle, individu et exigence d'universalité. Bien que la science juridique n'ait pas pour finalité la reconstitution d'événements passés, nous pouvons tout de même admettre que, pour agencer les faits de manière à faire surgir la norme juridique en tant que "corrélation à haute fréquence", le raisonnement juridique utilise une forme de cohérence causale spécifique, qui est la cohérence narrative.

Pour Nérhot, analysant les travaux de McCormick, ${ }^{24}$ la création de normes utilise les mêmes opérations rationnelles que l'analyse des actions humaines ou de la société. Il faut souligner: 25

21 Nérhot, précité n 1, 310.

22 Ibid, 300

23 Ricœur, précité n 12, 217.

24 N McCormick "Coherence in Legal Justification" dans A Peczenik, L Lindhal et G Van Roermund (dir) Theory of Legal Science: Proceedings of the Conference on Legal Theory and Philosophy of Science, Lund, Sweden, December 11-14,1983 (D Reidel Publishing Company, Dordrecht, 1984) 37. 
[L]'interaction entre des normes existantes et des traits observables de la vie sociale ... Les normes et les actions individuelles, ou l'ordre social, dans leur aspect observable sont les deux faces de la même pièce.

Autrement dit, la cohérence normative n'est pas d'une autre nature que la cohérence narrative utilisée par les historiens et les sociologues. La norme juridique n'est pas le produit d'un raisonnement spécifique, elle n'a aucune dimension transcendante, mais utilise le même type d'agencement causal que le récit historique et pourquoi pas que le récit en général: 26

Nous rencontrons ici le travail herméneutique ... . [C]e qui éventuellement sera repris, ce n'est pas la vérité quelconque d'une réalité extérieure à la règle, et qui viendrait rencontrer une règle préconstruite dont le sens, intrinsèque, s'imposerait à l'interprète, une rencontre dont naîtrait un code social objectif, rationnel et prévisible. Ce qui est repris c'est un modèle.

Ce terme de "modèle" que nous avons employé accompagné de l'adjectif "mathématique" mais que nous pouvons employer désormais avec l'adjectif "narratif", relève, si l'on en croit Lyotard, de la définition d'axiomatiques différentes à partir d'un même métalangage qui est la logique. Or, qu'estce qu'une logique axiomatique sinon le choix posé sans démonstration préalable de symboles, d'une syntaxe normative et d'opérations autorisées à partir de ces deux éléments? On peut donc créer une axiomatique mathématique tout comme une axiomatique narrative, à condition de respecter les contraintes logiques fondamentales définies par les logiciens. C'est ce qu'ont tenté de faire les sémioticiens, partant du principe qu'un système logique n'est pas obligatoirement mathématique et qu'il suffit qu'il satisfasse aux conditions de consistance, de complétude syntaxique, de décidabilité et d'indépendance des axiomes les uns par rapport aux autres. ${ }^{27}$

Le modèle narratif est donc le résultat d'une axiomatique mise en place par les sémioticiens pour rendre compte à la fois de l'invention littéraire et du récit historique. Le récit est désormais perçu comme ayant une grammaire propre qui s'enracine dans une grammaire de la relation sujet/prédicat. De plus, il est désormais convenu de distinguer l'immanence des structures narratives et l'apparence des structures linguistiques. Enfin les chercheurs partagent: ${ }^{28}$

[U]ne croyance en la possibilité d'articuler la suite des événements racontés en séquences d'actions dont certaines au moins pourraient prendre place dans un lexique universel de la narrativité; croyance en la possibilité de dégager les règles, elles-mêmes universelles, de combinaison de ces unités.

Ainsi, le savoir narratif serait-il obtenu à partir d'un modèle de compréhension parallèle au modèle mathématique, susceptible d'être décrit systématiquement dans ses symboles et dans ses

25 Nérhot, précité n 1, 310

26 Ibid, 329

27 Voir Lyotard, précité n 7, 69-78.

28 Claude Brémond Logique du récit (Seuil, Paris, 1973) 102. 
opérations. En fait, la plupart des chercheurs en sciences humaines et sociales sont réticents à accepter l'hypothèse d'un modèle qui couvrirait l'activité littéraire ainsi que le raisonnement historique, ethnologique ou juridique par exemple. Pourtant, le récit est un modèle dont le contenu peut être réel (récit historique) ou irréel (récit utopique par exemple) car il est un modèle de représentation pour la réalité. En effet, l'utopie est fonctionnelle et efficace, c'est à dire source de savoir sur le monde, dans la mesure où elle utilise un modèle subsumant qui est celui du récit, palimpseste que l'on peut retrouver dans tous les textes narratifs. La différence entre les narrations factuelle et fictionnelle ne réside donc pas dans la présence ou l'absence de fonction cognitive dans l'une ou l'autre. Celle-ci est en fait à l'œuvre dans tous les types de récit, grâce à la cohérence structurelle du modèle narratif.

L'une des premières analyses du modèle narratif a été celle faite par Claude Lévi-Strauss pour rendre compte de l'organisation du sens dans les récits mythiques, analyse achronique de "paquets de sens"29 présentant des univers axiologiques en opposition: "forme protocolaire d'organisation des contenus axiologiques contradictoires". ${ }^{30}$ Il y démontre comment dans les récits mythiques un questionnement sur les valeurs et les finalités de la vie humaine sont posées en termes d'oppositions conceptuelles. Une seconde analyse sémiotique a mis en évidence le rôle clef de la dimension temporelle dans le modèle du récit: ${ }^{31}$

[L]'épreuve, considérée comme expression figurative du modèle transformationnel, introduit une dimension diachronique qui, tout en opposant les contenus axiologiques investis dans les structures qui

la précèdent et qui la suivent, rend compte en même temps de leur transformation.

Cette lecture permettait de rendre compte de la façon dont la cohérence était recherchée à partir d'opérations effectuées sur les contenus sous la forme anthropomorphe d'une action sur le monde. Ainsi donc, l'épreuve (mise à l'épreuve d'un héros individuel, collectif ou idéel anthropomorphisé) ou plus exactement les épreuves constituent-elles la véritable grammaire narrative du récit, celle qui opère sur les contenus et qui n'est définie complètement ni par la pure succession temporelle, ni par un pur lien logique. Par ailleurs, cette grammaire à choix multiples maintient une liberté d'organisation des contenus. ${ }^{32}$

Enfin, A J Greimas a tenté d'unifier ces deux approches du récit, par les contenus axiologiques ou par la grammaire narrative, dans l'élaboration d'une figure géométrique dénommée carré sémiotique qui résumerait les contenus axiologiques posés, ainsi que les opérations de transformation qui s'y appliquent. Celles-ci permettent d'aboutir, grâce à leur mise en œuvre par les

29 Claude Lévi-Strauss Anthropologie structurale (Plon, Paris, 1958) 230.

30 Ibid, 248.

31 A J Greimas Sémantique structurale (Seuil, Paris, 1986) 212.

32 Ibid, 211 
actants des contenus, à un nouvel ordre axiologique ou au même ordre validé (récits de l'ordre présent refusé, récits de l'ordre présent accepté). Ce carré sémiotique se présente ainsi: sur le côté supérieur du carré deux contenus axiologiques ( $\mathrm{S} 1$ et $\mathrm{S} 2$ qui entretiennent une relation de contrariété), sur le côté inférieur du carré deux contenus (non S2 et non S1 qui entretiennent une relation de contradiction entre eux ainsi qu'une relation de présupposition respectivement avec S2 et S1). Les opérations narratives de transformation s'effectueraient le long des quatre côtés du carré ainsi que de ses diagonales vectorisées: ${ }^{33}$

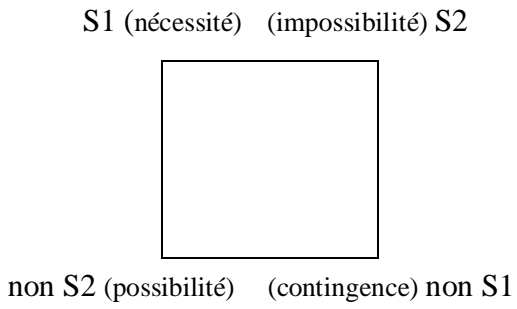

Ainsi donc arrive-t-on à concevoir un modèle du récit qui n'est ni les événements racontés, ni le discours qui les racontent mais une série d'opérations logiques effectuées sur des valeurs posées au départ, dont on envisage les contradictions ou les conditions d'acceptabilité. Si l'on adhère à cette définition du récit en tant que structure de la signification, modèle logique, on peut très bien adopter l'idée de son utilisation inconsciente par les juristes non seulement dans la formation de la jurisprudence (application de la norme à des cas) mais aussi dans la formation de la norme même. Car le récit ne dit pas uniquement l'histoire d'un individu dans la société, comme il le fait certes dans le roman, mais de manière plus large, il permet de mimer les jeux logiques sur des valeurs utilisées par la praxis humaine et ceci y compris dans le récit de fiction. Si l'on accepte l'idée que le récit n'est pas uniquement, comme le croit le sens commun, une fiction, produit de limagination (Littérature) ou encore la simple relation de faits (Histoire) mais un modèle du savoir, sa pertinence en Droit ne fait plus vraiment problème. La cohérence narrative devient, comme le soutient Nérhot, une dimension essentielle du raisonnement juridique.

\section{CONCLUSION}

Au terme de cette analyse nous ferons deux constatations. La première est que les grands Récits de légitimation qui soustendent le raisonnement en sciences humaines et sociales perdent de plus en plus de leur pertinence dans le monde contemporain. Jean Bessière souligne que dans un contexte de mondialisation "aucune accentuation anthropologique seule, ni aucune identité culturelle seule ne

33 Ceci est un exemple très simplifié de carré sémiotique. 
sauraient constituer, représenter, figurer un cadre englobant de l'existence collective". ${ }^{34}$ Il précise que naguère en Occident en tout cas: ${ }^{35}$

Tout temps et toute histoire sont contaminés par une anthropologie de l'histoire qui est la sécularisation d'une vision chrétienne de l'histoire. ... [L]'histoire est une crise, elle est un progrès, c'est-à-dire la possibilité de la réalisation d'une attente.

La deuxième constatation est que les récits littéraires contemporains se prévalant de ces constats mettent fortement en cause la cohérence narrative jusqu'ici extrêmement liée à la culture occidentale, comme nous avons tenté de le montrer.

De ces constatations, il faudrait conclure que ce modèle des savoirs qu'est le récit ne correspond plus aux sociétés contemporaines. Donc, si notre démonstration ci-dessus a quelque pertinence, nul doute que cela entraînerait une redéfinition de tout ce que Lyotard, dans son ouvrage La Condition postmoderne, appelait les savoirs narratifs, c'est-à-dire en gros les sciences humaines et sociales. Nous pouvons observer les prémisses de cette redéfinition sous sa forme négative: la mise en cause des normes et des principes que nous qualifiions naguère d'universels et qui se révèlent aux yeux d'une partie non négligeable de l'humanité relatifs et culturels.

34 Jean Bessière Le Roman contemporain (PUF, Paris, 2010) 95.

35 Ibid, 259 
